\title{
Precision Measurement of the Boron to Carbon Flux Ratio in Cosmic Rays from 1.9 GV to 2.6 TV with the Alpha Magnetic Spectrometer on the International Space Station
}

M. Aguilar, ${ }^{26}$ L. Ali Cavasonza, ${ }^{1}$ G. Ambrosi,${ }^{32}$ L. Arruda,${ }^{24}$ N. Attig, ${ }^{21}$ S. Aupetit, ${ }^{17}$ P. Azzarello, ${ }^{16}$ A. Bachlechner, ${ }^{1}$ F. Barao, ${ }^{24}$ A. Barrau, ${ }^{17}$ L. Barrin, ${ }^{15}$ A. Bartoloni,${ }^{37}$ L. Basara, ${ }^{35}$ S. Başeğmez-du Pree, ${ }^{6}$ M. Battarbee, ${ }^{45}$ R. Battiston, ${ }^{35,36, a}$ U. Becker, ${ }^{9}$ M. Behlmann, ${ }^{9}$ B. Beischer, ${ }^{1}$ J. Berdugo, ${ }^{26}$ B. Bertucci, ${ }^{32,33}$ K. F. Bindel, ${ }^{22}$ V. Bindi, ${ }^{19}$ G. Boella, ${ }^{28,29}$ W. de Boer, ${ }^{22}$ K. Bollweg, ${ }^{20}$ V. Bonnivard,${ }^{17}$ B. Borgia,${ }^{37,38}$ M. J. Boschini ${ }^{28}$ M. Bourquin, ${ }^{16}$ E. F. Bueno,${ }^{39}$ J. Burger, ${ }^{9}$ F. Cadoux,${ }^{16}$ X. D. Cai, ${ }^{9}$ M. Capell,,${ }^{9}$ S. Caroff, ${ }^{3}$ J. Casaus,${ }^{26}$ G. Castellini, ${ }^{14}$ F. Cervelli, ${ }^{34}$ M. J. Chae, ${ }^{40}$ Y. H. Chang, ${ }^{10}$ A. I. Chen, ${ }^{9}$ G. M. Chen, ${ }^{6}$ H. S. Chen, ${ }^{6}$ L. Cheng, ${ }^{41}$ H. Y. Chou, ${ }^{10}$ E. Choumilov, ${ }^{9}$ V. Choutko, ${ }^{9}$ C. H. Chung, ${ }^{1}$ C. Clark, ${ }^{20}$ R. Clavero, ${ }^{23}$ G. Coignet, ${ }^{3}$ C. Consolandi, ${ }^{19}$ A. Contin, ${ }^{7,8}$ C. Corti, ${ }^{19}$ W. Creus,${ }^{44}$ M. Crispoltoni,,${ }^{32,33,15}$ Z. Cui, ${ }^{41}$ Y. M. Dai, ${ }^{5}$ C. Delgado, ${ }^{26}$ S. Della Torre ${ }^{28}$ O. Demakov, ${ }^{9}$ M. B. Demirköz, ${ }^{2}$ L. Derome,${ }^{17}$ S. Di Falco,${ }^{34}$ F. Dimiccoli,${ }^{35,36}$ C. Díaz,${ }^{26}$ P. von Doetinchem, ${ }^{19}$ F. Dong, ${ }^{30}$ F. Donnini, ${ }^{32,33,15}$ M. Duranti, ${ }^{32,33}$ D. D’Urso, ${ }^{32, b}$ A. Egorov, ${ }^{9}$ A. Eline, ${ }^{9}$ T. Eronen, ${ }^{45}$ J. Feng, ${ }^{44, c}$ E. Fiandrini, ${ }^{32,33}$ E. Finch,${ }^{31}$ P. Fisher, ${ }^{9}$ V. Formato, ${ }^{32,15}$ Y. Galaktionov, ${ }^{9}$ G. Gallucci, ${ }^{34}$ B. García, ${ }^{26}$ R. J. García-López, ${ }^{23}$ C. Gargiulo, ${ }^{15}$ H. Gast, ${ }^{1}$ I. Gebauer, ${ }^{22}$ M. Gervasi, ${ }^{28,29}$ A. Ghelfi, ${ }^{17}$ F. Giovacchini, ${ }^{26}$ P. Goglov, ${ }^{9}$ D. M. Gómez-Coral, ${ }^{27}$ J. Gong, ${ }^{30}$ C. Goy, ${ }^{3}$ V. Grabski, ${ }^{27}$ D. Grandi, ${ }^{28}$ M. Graziani, ${ }^{32,33}$ K. H. Guo, ${ }^{18}$ S. Haino, ${ }^{44}$ K. C. Han ${ }^{25}$ Z. H. He, ${ }^{18}$ M. Heil,${ }^{9}$ J. Hoffman, ${ }^{19}$ T. H. Hsieh, ${ }^{9}$ H. Huang, ${ }^{44, d}$ Z. C. Huang, ${ }^{18}$ C. Huh, ${ }^{13}$ M. Incagli, ${ }^{34}$ M. Ionica, ${ }^{32}$ W. Y. Jang, ${ }^{13}$ H. Jinchi, ${ }^{25}$ S. C. Kang, ${ }^{13}$ K. Kanishev, ${ }^{35,36}$ G. N. Kim, ${ }^{13}$ K. S. Kim, ${ }^{13}$ Th. Kirn, ${ }^{1}$ C. Konak, ${ }^{2}$ O. Kounina, ${ }^{9}$ A. Kounine, ${ }^{9}$ V. Koutsenko, ${ }^{9}$ M. S. Krafczyk, ${ }^{9}$ G. La Vacca, ${ }^{28}$ E. Laudi, ${ }^{15}$ G. Laurenti, ${ }^{7}$ I. Lazzizzera, ${ }^{35,36}$ A. Lebedev, ${ }^{9}$ H. T. Lee, ${ }^{43}$ S. C. Lee, ${ }^{44}$ C. Leluc, ${ }^{16}$ H. S. Li ${ }^{42}$ J. Q. Li ${ }^{9, e}$ J. Q. Li,${ }^{30}$ Q. Li, ${ }^{30}$ T. X. Li, ${ }^{18}$ W. Li, ${ }^{4}$ Y. Li, ${ }^{16, c}$ Z. H. Li, ${ }^{6}$ Z. Y. Li, ${ }^{44, c}$ S. Lim, ${ }^{13}$ C. H. Lin ${ }^{44}$ P. Lipari, ${ }^{37}$ T. Lippert, ${ }^{21}$ D. Liu, ${ }^{10}$ Hu Liu, ${ }^{26, f}$ V. D. Lordello, ${ }^{39}$ S. Q. Lu, ${ }^{44, c}$ Y. S. Lu, ${ }^{6}$ K. Luebelsmeyer, ${ }^{1}$ F. Luo, ${ }^{41}$ J. Z. Luo, ${ }^{30}$ S. S. Lv, ${ }^{18}$ F. Machate, ${ }^{1}$ R. Majka, ${ }^{31}$ C. Mañá, ${ }^{26}$ J. Marín, ${ }^{26}$ T. Martin,${ }^{20}$ G. Martínez, ${ }^{26}$ N. Masi, ${ }^{7}$ D. Maurin, ${ }^{17}$ A. Menchaca-Rocha, ${ }^{27}$ Q. Meng, ${ }^{30}$ V. M. Mikuni, ${ }^{39}$ D. C. Mo, ${ }^{18}$ L. Morescalchi, ${ }^{34, g}$ P. Mott ${ }^{20}$ T. Nelson, ${ }^{19}$ J. Q. Ni ${ }^{18}$ N. Nikonov, ${ }^{1}$ F. Nozzoli, ${ }^{32, b}$ A. Oliva ${ }^{26}$ M. Orcinha, ${ }^{24}$ F. Palmonari, ${ }^{7,8}$ C. Palomares,${ }^{26}$ M. Paniccia,${ }^{16}$ M. Pauluzzi, ${ }^{32,33}$ S. Pensotti, ${ }^{28,29}$ R. Pereira, ${ }^{19}$ N. Picot-Clemente, ${ }^{12}$ F. Pilo,${ }^{34}$ C. Pizzolotto, ${ }^{32, b}$ V. Plyaskin, ${ }^{9}$

M. Pohl,${ }^{16}$ V. Poireau, ${ }^{3}$ A. Putze, ${ }^{3, h}$ L. Quadrani, ${ }^{7,8}$ X. M. Qi ${ }^{18}$ X. Qin, ${ }^{32, i}$ Z. Y. Qu, ${ }^{44, j}$ T. Räihä, ${ }^{1}$ P. G. Rancoita, ${ }^{28}$ D. Rapin, ${ }^{16}$ J. S. Ricol, ${ }^{17}$ S. Rosier-Lees,${ }^{3}$ A. Rozhkov, ${ }^{9}$ D. Rozza, ${ }^{28}$ R. Sagdeev,${ }^{11}$ J. Sandweiss, ${ }^{31}$ P. Saouter, ${ }^{16}$ S. Schael, ${ }^{1}$ S. M. Schmidt, ${ }^{21}$ A. Schulz von Dratzig, ${ }^{1}$ G. Schwering, ${ }^{1}$ E. S. Seo, ${ }^{12}$ B. S. Shan, ${ }^{4}$ J. Y. Shi, ${ }^{30}$ T. Siedenburg, ${ }^{1}$ D. Son, ${ }^{13}$ J. W. Song, ${ }^{41}$ W. H. Sun, ${ }^{9, k}$ M. Tacconi, ${ }^{28}$ X. W. Tang, ${ }^{6}$ Z. C. Tang, ${ }^{6}$ L. Tao, ${ }^{3}$ D. Tescaro, ${ }^{23}$ Samuel C. C. Ting, ${ }^{9,15}$

S. M. Ting, ${ }^{9}$ N. Tomassetti, ${ }^{32,33}$ J. Torsti, ${ }^{45}$ C. Türkoğlu, ${ }^{2}$ T. Urban, ${ }^{20}$ V. Vagelli, ${ }^{32,33}$ E. Valente, ${ }^{37,38}$ C. Vannini, ${ }^{34}$

E. Valtonen, ${ }^{45}$ M. Vázquez Acosta, ${ }^{23}$ M. Vecchi, ${ }^{39}$ M. Velasco, ${ }^{26}$ J. P. Vialle, ${ }^{3}$ V. Vitale, ${ }^{32, b}$ S. Vitillo, ${ }^{16}$ L. Q. Wang, ${ }^{41}$ N. H. Wang, ${ }^{41}$ Q. L. Wang, ${ }^{5}$ X. Wang, ${ }^{9}$ X. Q. Wang, ${ }^{6}$ Z. X. Wang, ${ }^{18}$ C. C. Wei, ${ }^{44,1}$ Z. L. Weng, ${ }^{9}$ K. Whitman, ${ }^{19}$

J. Wienkenhöver, ${ }^{1}$ H. Wu, ${ }^{30}$ X. Wu, ${ }^{16}$ X. Xia, ${ }^{26, i}$ R. Q. Xiong, ${ }^{30}$ W. Xu, ${ }^{9}$ Q. Yan, ${ }^{9}$ J. Yang, ${ }^{40}$ M. Yang, ${ }^{6}$ Y. Yang, ${ }^{42}$ H. Yi, ${ }^{30}$ Y. J. Yu, ${ }^{5}$ Z. Q. Yu, ${ }^{6}$ S. Zeissler, ${ }^{22}$ C. Zhang, ${ }^{6}$ J. Zhang, ${ }^{9}$ d J. H. Zhang, ${ }^{30}$ S. D. Zhang, ${ }^{9, e}$ S. W. Zhang, ${ }^{6}$ Z. Zhang, Z. M. Zheng, ${ }^{4}$ Z. Q. Zhu, ${ }^{9, m}$ H. L. Zhuang, ${ }^{6}$ V. Zhukov, ${ }^{1}$ A. Zichichi, ${ }^{7,8}$ N. Zimmermann, ${ }^{1}$ and P. Zuccon ${ }^{9}$

(AMS Collaboration)

\author{
${ }^{1}$ I. Physics Institute and JARA-FAME, RWTH Aachen University, D-52056 Aachen, Germany \\ ${ }^{2}$ Department of Physics, Middle East Technical University (METU), 06800 Ankara, Turkey \\ ${ }^{3}$ Laboratoire d'Annecy-le-Vieux de Physique des Particules (LAPP), CNRS/IN2P3 and Université Savoie Mont Blanc, \\ F-74941 Annecy-le-Vieux, France \\ ${ }^{4}$ Beihang University (BUAA), Beijing 100191, China \\ ${ }^{5}$ Institute of Electrical Engineering (IEE), Chinese Academy of Sciences, Beijing 100190, China \\ ${ }^{6}$ Institute of High Energy Physics (IHEP), Chinese Academy of Sciences, Beijing 100049, China \\ ${ }^{7}$ INFN Sezione di Bologna, I-40126 Bologna, Italy \\ ${ }^{8}$ Università di Bologna, I-40126 Bologna, Italy \\ ${ }^{9}$ Massachusetts Institute of Technology (MIT), Cambridge, Massachusetts 02139, USA \\ ${ }^{10}$ National Central University (NCU), Chung-Li, Tao Yuan 32054, Taiwan \\ ${ }^{11}$ East-West Center for Space Science, University of Maryland, College Park, Maryland 20742, USA \\ ${ }^{12}$ IPST, University of Maryland, College Park, Maryland 20742, USA \\ ${ }^{13}$ CHEP, Kyungpook National University, 41566 Daegu, Korea \\ ${ }^{14}$ CNR-IROE, I-50125 Firenze, Italy
}




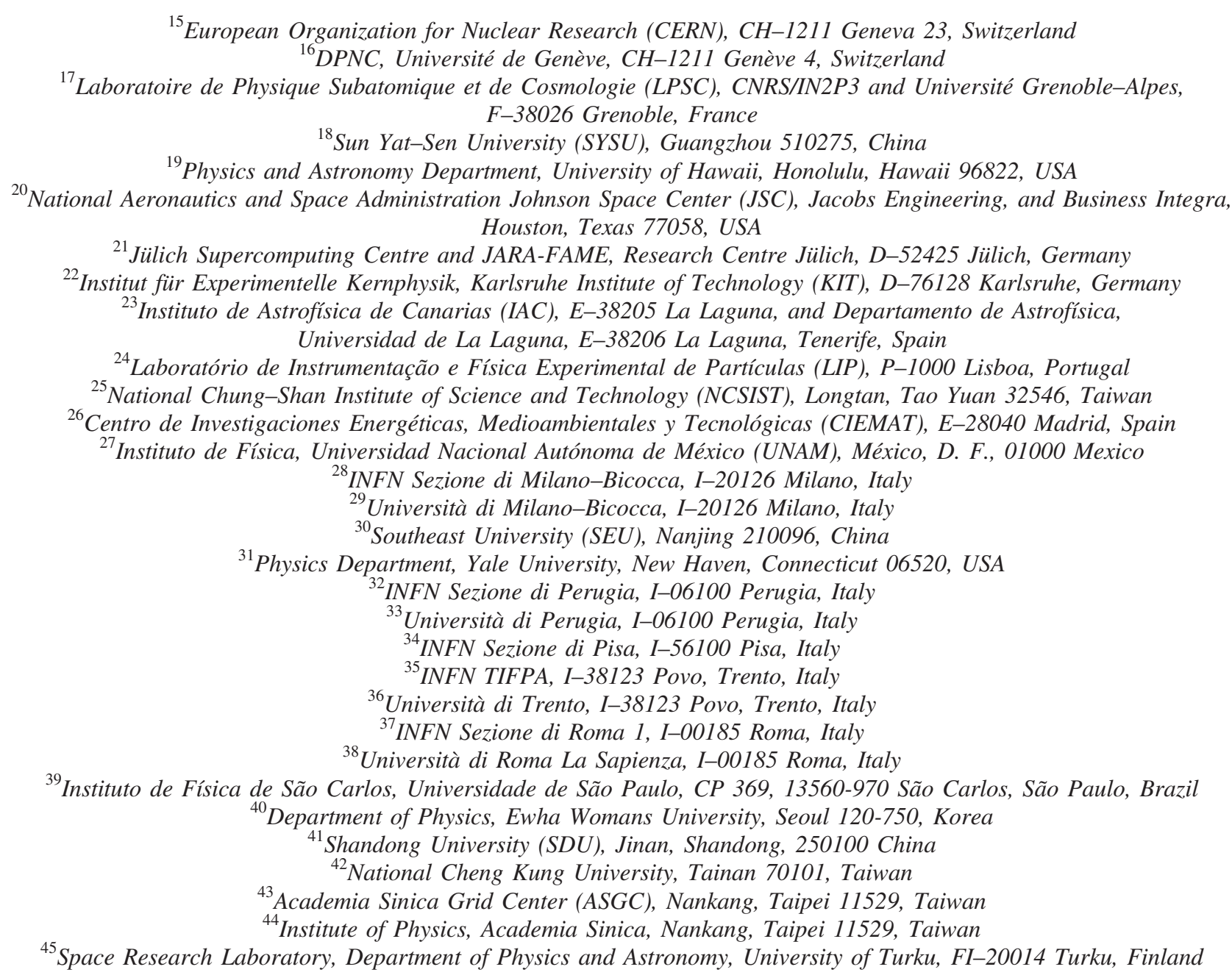

(Received 5 October 2016; published 28 November 2016)

Knowledge of the rigidity dependence of the boron to carbon flux ratio $(\mathrm{B} / \mathrm{C})$ is important in understanding the propagation of cosmic rays. The precise measurement of the $\mathrm{B} / \mathrm{C}$ ratio from $1.9 \mathrm{GV}$ to 2.6 TV, based on 2.3 million boron and 8.3 million carbon nuclei collected by AMS during the first 5 years of operation, is presented. The detailed variation with rigidity of the $\mathrm{B} / \mathrm{C}$ spectral index is reported for the first time. The $\mathrm{B} / \mathrm{C}$ ratio does not show any significant structures in contrast to many cosmic ray models that require such structures at high rigidities. Remarkably, above $65 \mathrm{GV}$, the $\mathrm{B} / \mathrm{C}$ ratio is well described by a single power law $R^{\Delta}$ with index $\Delta=-0.333 \pm 0.014$ (fit) \pm 0.005 (syst), in good agreement with the Kolmogorov theory of turbulence which predicts $\Delta=-1 / 3$ asymptotically.

DOI: 10.1103/PhysRevLett.117.231102

Carbon nuclei in cosmic rays are thought to be mainly produced and accelerated in astrophysical sources, while boron nuclei are entirely produced by the collision of heavier nuclei, such as carbon and oxygen, with nuclei of the interstellar matter. Therefore, the boron to carbon

Published by the American Physical Society under the terms of the Creative Commons Attribution 3.0 License. Further distribution of this work must maintain attribution to the author(s) and the published article's title, journal citation, and DOI. flux ratio $(\mathrm{B} / \mathrm{C})$ directly measures the average amount of interstellar material traversed by cosmic rays [1].

In cosmic ray propagation models, where cosmic rays are described as a relativistic gas scattering on a magnetized plasma [1], the $\mathrm{B} / \mathrm{C}$ ratio is used to constrain the spatial diffusion coefficient $D$, as the $\mathrm{B} / \mathrm{C}$ ratio is proportional to $1 / D$ at high rigidities $R$. The diffusion coefficient dependence on rigidity is $D \propto R^{-\delta}$, where $\delta$ is predicted to be $\delta=-1 / 3$ with the Kolmogorov theory of interstellar turbulence [2], or $\delta=-1 / 2$ using the Kraichnan theory [3]. The measured B/C spectral index $\Delta$, obtained from a fit at high rigidities of the 
$(\mathrm{B} / \mathrm{C}) \propto R^{\Delta}$, approaches the diffusion spectral index $\delta$ asymptotically $(\Delta=\delta)$.

Precise measurements of proton and helium fluxes by Alpha Magnetic Spectrometer (AMS) [4,5] have led to the development of models predicting a deviation from a single power law of the $\mathrm{B} / \mathrm{C}$ ratio spectrum at high rigidity [6]. Independently, a deviation from single power law of the $\mathrm{B} / \mathrm{C}$ ratio at high rigidities is necessary in models [7-9] that explain the AMS results on positron fraction [10] and antiprotons [11].

Because of the importance of the $\mathrm{B} / \mathrm{C}$ ratio to the understanding of cosmic rays, over the last 30 years there have been many measurements [12-21]. Typically, these measurements have errors larger than $15 \%$ at $100 \mathrm{GV}$.

In this Letter we report the precise measurement of the $\mathrm{B} / \mathrm{C}$ ratio in cosmic rays in the rigidity range from $1.9 \mathrm{GV}$ to $2.6 \mathrm{TV}$ based on data collected by AMS during the first 5 years (May 19, 2011 to May 26, 2016) of operation onboard the International Space Station (ISS). In this measurement the total error is $\sim 3 \%$ at $100 \mathrm{GV}$.

Detector.-The layout and description of the AMS detector are presented in Ref. [22]. The key elements used in this measurement are the permanent magnet [23], the silicon tracker, four planes of time of flight (TOF) scintillation counters, and an array of 16 anticoincidence counters. AMS also contains a transition radiation detector (TRD), a ring imaging Čerenkov detector (RICH), and an electromagnetic calorimeter (ECAL).

The tracker [24] has nine layers, the first $(L 1)$ at the top of the detector, the second ( $L 2)$ above the magnet, six ( $L 3$ to $L 8$ ) within the bore of the magnet, and the last $(L 9)$ above the ECAL. $L 2$ to $L 8$ constitute the inner tracker.

Together, the tracker and the magnet measure the rigidity $R$ of charged cosmic rays. The spatial resolution in each tracker layer is $8 \mu \mathrm{m}$ on average in the bending direction for boron and $10 \mu \mathrm{m}$ for carbon. The resulting maximum detectable rigidities (MDRs) are 3.0 TV for boron and 2.6 TV for carbon over the $3 \mathrm{~m}$ lever arm from $L 1$ to $L 9$. Each layer of the tracker provides also an independent measurement of the charge $Z$ with a resolution of $\Delta Z / Z=5 \%$ for both boron and carbon. Overall the inner tracker has a resolution of $\Delta Z / Z=2 \%$ for boron and carbon.

Two of the TOF planes [25] are located above the magnet (upper TOF) and the other two planes are located below the magnet (lower TOF). The overall velocity $(\beta=v / c)$ resolution has been measured to be $\Delta \beta / \beta^{2}=0.01$ for boron and carbon nuclei. This discriminates between upward- and downward-going particles. The pulse heights of the two upper planes are combined to provide an independent measurement of the charge with an accuracy $\Delta Z / Z=3 \%$ for both boron and carbon. The pulse heights from the two lower planes are combined to provide another independent charge measurement with the same accuracy.
Boron and carbon nuclei traversing AMS were triggered as described in detail in Ref. [5]. The trigger efficiencies for both nuclei were measured to be $>98 \%$ over the entire rigidity range.

Monte Carlo (MC) simulated events were produced using a dedicated program developed by the collaboration based on the GEANT-4.10.1 package [26]. The program simulates electromagnetic and hadronic interactions of particles in the material of AMS and generates detector responses. The Glauber-Gribov model was used for the description of the inelastic cross sections. The INCL++ package [27] was used to model nucleus-nucleus inelastic interactions below $5 \mathrm{GeV} / \mathrm{n}$ and the DPMJET-II.5 package [28] was used at higher energies. The nucleus-nucleus hadronic elastic and quasielastic scatterings were treated using the model developed for helium nuclei [5] and validated by detailed comparison between data and simulation.

Event selection.-In the first 5 years AMS has collected $8.5 \times 10^{10}$ cosmic ray events. The collection time used in this analysis includes only those seconds during which the detector was in normal operating conditions and, in addition, AMS was pointing within $40^{\circ}$ of the local zenith and the ISS was outside of the South Atlantic Anomaly. Because of the influence of the geomagnetic field, this collection time for galactic cosmic rays increases with rigidity becoming constant at $1.23 \times 10^{8} \mathrm{sec}$ above $30 \mathrm{GV}$.

Events are required to be downward going and to have a reconstructed track in the inner tracker and passing through $L 1$. In the highest rigidity region, $R \geq 0.88 \mathrm{TV}$, the track is also required to pass through $L 9$. Track fitting quality criteria such as a $\chi^{2} /$ d.o.f. $<10$ in the bending coordinate are applied, similar to Refs. $[4,5]$.

The measured rigidity is required to be greater than a factor of 1.2 times the maximum geomagnetic cutoff within the AMS field of view. The cutoff was calculated by backtracing [29] particles from the top of AMS out to 50 Earth's radii using the most recent IGRF [30] geomagnetic model.

Charge measurements on tracker $L 1$, inner tracker, upper TOF, lower TOF, and, for $R>0.88 \mathrm{TV}$, tracker $L 9$ are required to be compatible with charge $Z=5$ for boron, and $Z=6$ for carbon, as shown in Fig. 1 of the Supplemental Material [31] for the inner tracker. This selection yields purities of $90 \%$ to $95 \%$ depending on rigidity for boron, and $99 \%$ for carbon.

The residual background to boron and carbon events resulting from interactions of heavy nuclei such as carbon, nitrogen, and oxygen in the material between $L 1$ and $L 2$ (TRD and upper TOF) is evaluated by fitting the charge distribution of tracker $L 1$ with charge distribution templates of B, C, N, and O as shown in Fig. 2 of the Supplemental Material [31]. The charge distribution templates are obtained from a selection of noninteracting samples on $L 2$ by the use of the charge measurement with $L 1$ 


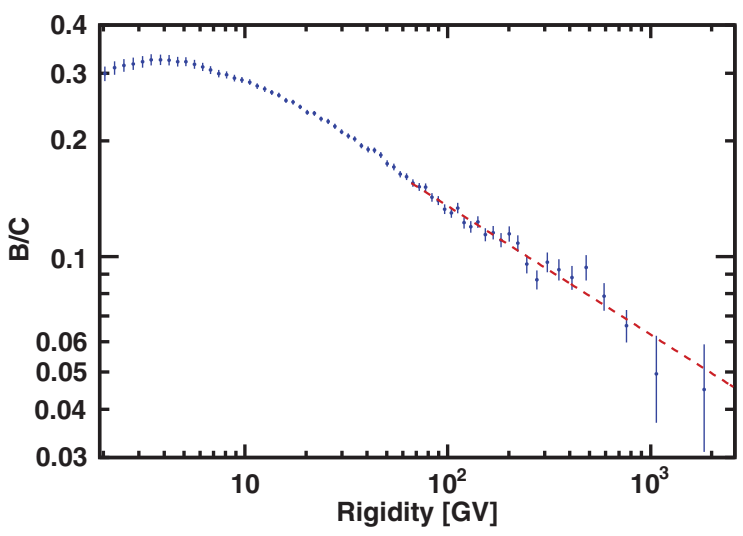

FIG. 1. The AMS boron to carbon ratio $(\mathrm{B} / \mathrm{C})$ as a function of rigidity in the interval from $1.9 \mathrm{GV}$ to $2.6 \mathrm{TV}$ based on 2.3 million boron and 8.3 million carbon nuclei. The dashed line shows the single power law fit starting from $65 \mathrm{GV}$ with index $\Delta=$ $-0.333 \pm 0.014$ (fit) \pm 0.005 (syst).

and $L 3-L 8$. This residual background is $<3 \%$ for the boron sample and $<0.5 \%$ for carbon.

The background from carbon, nitrogen, and oxygen interactions on materials above $L 1$ (thin support structures made by carbon fiber and aluminum honeycomb) has been estimated from simulation, using MC samples generated according to AMS flux measurements [32]. The simulation of nuclear interactions has been validated using data as shown in Fig. 3 of the Supplemental Material [31]. The background from interactions above $L 1$ in the boron sample is $2 \%$ at $2 \mathrm{GV}$ and increases up to $8 \%$ at 2.6 $\mathrm{TV}$, while for the carbon sample it is $<0.5 \%$ over the entire rigidity range. The total correction to the $\mathrm{B} / \mathrm{C}$ ratio from background subtraction is $-2 \%$ at $2 \mathrm{GV},-3 \%$ at $20 \mathrm{GV}$, $-7 \%$ at $200 \mathrm{GV}$, and $-10 \%$ at $2 \mathrm{TV}$.

After background subtraction the sample contains $2.3 \times 10^{6}$ boron and $8.3 \times 10^{6}$ carbon nuclei.

Data analysis.-The isotropic flux $\Phi_{i}^{Z}$ for nuclei of charge $Z$ in the $i$ th rigidity bin $\left(R_{i}, R_{i}+\Delta R_{i}\right)$ is given by

$$
\Phi_{i}^{Z}=\frac{N_{i}^{Z}}{A_{i}^{Z} \epsilon_{i}^{Z} T_{i} \Delta R_{i}},
$$

where $N_{i}^{Z}$ is the number of events of charge $Z$ corrected for bin-to-bin migrations, $A_{i}^{Z}$ is the effective acceptance, $\epsilon_{i}^{Z}$ is the trigger efficiency, and $T_{i}$ is the collection time. The $\mathrm{B} / \mathrm{C}$ ratio in each rigidity bin is then given by

$$
\left(\frac{\mathrm{B}}{\mathrm{C}}\right)_{i}=\frac{\Phi_{i}^{\mathrm{B}}}{\Phi_{i}^{\mathrm{C}}}=\frac{N_{i}^{\mathrm{B}}}{N_{i}^{\mathrm{C}}}\left(\frac{A_{i}^{\mathrm{B}}}{A_{i}^{\mathrm{C}}} \frac{\epsilon_{i}^{\mathrm{B}}}{\epsilon_{i}^{\mathrm{C}}}\right)^{-1} .
$$

In this Letter the $\mathrm{B} / \mathrm{C}$ ratio was measured in 67 bins from $1.9 \mathrm{GV}$ to $2.6 \mathrm{TV}$ with bin widths chosen according to the rigidity resolution.

The bin-to-bin migration of events was corrected using the unfolding procedure described in Ref. [4]

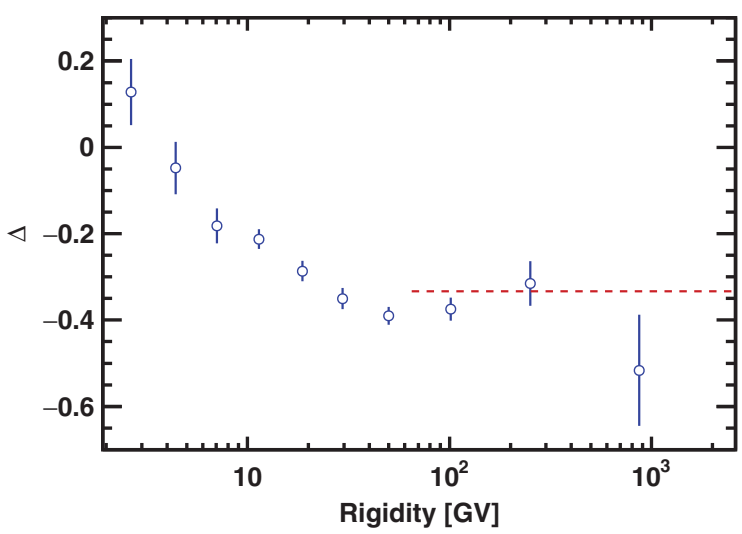

FIG. 2. The $\mathrm{B} / \mathrm{C}$ spectral index $\Delta$ as a function of rigidity. The dashed red line shows the single power law fit result to the $\mathrm{B} / \mathrm{C}$ ratio above $65 \mathrm{GV}$; see Fig. 1 .

independently for the boron and the carbon samples. This results in a correction on the $\mathrm{B} / \mathrm{C}$ ratio of $-2.4 \%$ at $2 \mathrm{GV},-0.5 \%$ at $20 \mathrm{GV},-5 \%$ at $200 \mathrm{GV}$, and $-13 \%$ at $2 \mathrm{TV}$.

Extensive studies were made of the systematic errors. These errors include the uncertainties in the two background estimations discussed above, in the trigger efficiency, in the acceptance calculation, in the rigidity resolution function, and in the absolute rigidity scale.

The systematic error on the $\mathrm{B} / \mathrm{C}$ ratio associated with background subtraction is dominated by the uncertainty of $\sim 10 \%$ in the boron sample background estimation for interactions above $L 1$, see, for example, Fig. 3 of the Supplemental Material [31]. The total background subtraction error on the $\mathrm{B} / \mathrm{C}$ ratio is $<1 \%$ over the entire rigidity range.

The systematic error on the $\mathrm{B} / \mathrm{C}$ ratio associated with the trigger efficiency is $<0.5 \%$ over the entire rigidity range.

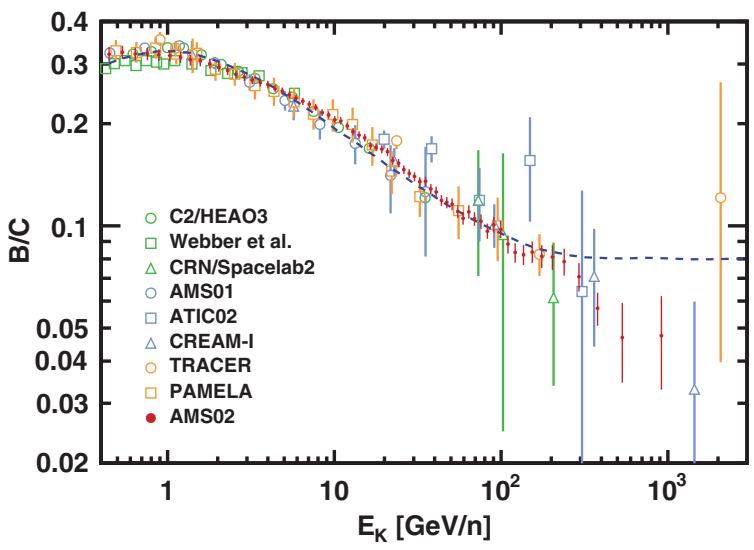

FIG. 3. The boron to carbon ratio as a function of kinetic energy per nucleon $E_{K}$ compared with measurements since the year 1980 [12-21]. The dashed line is the $\mathrm{B} / \mathrm{C}$ ratio required for the model of Ref. [7]. 
The effective acceptances $A_{i}^{Z}$ were calculated using MC simulation, and corrected for small differences between the data and MC calculations related to (a) event reconstruction and selection, namely, in the efficiencies of velocity determination, track finding, charge determination, and tracker quality cuts and (b) the inelastic interactions in the AMS materials. The total correction to the acceptance term $A_{i}^{\mathrm{B}} / A_{i}^{\mathrm{C}}$ was found to be $<5 \%$ over the entire rigidity range.

The systematic error on the $\mathrm{B} / \mathrm{C}$ ratio associated with the reconstruction and selection is $<1 \%$ over the entire rigidity range.

The material traversed by nuclei between $L 1$ and $L 9$ is composed primarily of carbon and aluminum, as described in detail in Ref. [5]. The corresponding inelastic cross sections for $\mathrm{C}+\mathrm{C}$ and $\mathrm{C}+\mathrm{Al}$ have only been measured below $10 \mathrm{GV}$ [33], and there is no data for $\mathrm{B}+\mathrm{C}$ and $\mathrm{B}+\mathrm{Al}$. To verify the MC predictions, boron and carbon event samples that traverse materials between $L 8$ and $L 9$ (Lower TOF and RICH) without interacting are measured in data and compared with MC calculations with GlauberGribov inelastic cross sections varied within $\pm 10 \%$. The resulting cross sections with the best agreement to data above $30 \mathrm{GV}$ were chosen. Figure 4 of the Supplemental Material [31] shows the measured survival probabilities between $L 8$ and $L 9$ compared with the simulation for boron and carbon. The survival probability between $L 1$ and $L 2$ has been calculated using data periods in which AMS was horizontal, i.e., $\sim 90^{\circ}$ with respect to the zenith [5]. This independently verifies the inelastic cross sections. The systematic error on the $\mathrm{B} / \mathrm{C}$ ratio from the ratio of the acceptances due to uncertainties of inelastic cross sections is evaluated to be $3 \%$ at $4 \mathrm{GV}$ decreasing to $<2 \%$ above $10 \mathrm{GV}$.

An additional systematic error on the $\mathrm{B} / \mathrm{C}$ ratio is due to the difference in the interaction of ${ }^{10} \mathrm{~B}$ and ${ }^{11} \mathrm{~B}$ within the detector material. This error is $<0.3 \%$ over the entire rigidity range, and was obtained assuming an isotopic composition of $Y_{\mathrm{B}}={ }^{11} \mathrm{~B} /\left({ }^{10} \mathrm{~B}+{ }^{11} \mathrm{~B}\right)=0.7 \pm 0.1$ for boron and pure ${ }^{12} \mathrm{C}$ for carbon. These compositions have been measured by AMS between 1 to $10 \mathrm{GeV} / \mathrm{n}$ by comparing fluxes derived in rigidity using the tracker with the fluxes measured in kinetic energy using TOF and RICH [32].

The rigidity resolution functions $\Delta(1 / R)$ for boron and carbon are similar to that of helium discussed in Ref. [5]. They have a pronounced Gaussian core characterized by width $\sigma$ and non-Gaussian tails more than $2.5 \sigma$ away from the center. The resolution functions have been verified with four procedures. First, the differences of the coordinates measured in $L 3$ or $L 5$ to those obtained from the track fit using the measurements from $L 1, L 2, L 4, L 6, L 7$, and $L 8$ were compared between data and simulation. This procedure directly measures the tracker bending coordinate accuracy of $8 \mu \mathrm{m}$ for boron and $10 \mu \mathrm{m}$ carbon, as shown in
Fig. 5 of the Supplemental Material [31]. Similar results were obtained for the rest of the inner tracker layers. Second, the distribution of the scattering angle, defined as the difference between the inner tracker track and the $L 1$ to $L 2$ trajectory, was compared between data and simulation as shown in Fig. 6 of the Supplemental Material [31] for carbon, and found to be in good agreement. This comparison verifies the multiple, nucleus-nucleus elastic, and quasielastic scatterings. Third, in order to validate the alignment of the external layers $L 1$ and $L 9$, the difference between the rigidities measured using the information from $L 1$ to $L 8$ and from $L 2$ to $L 9$ were compared between data and the simulation and found to be in good agreement similar to Ref. [4]. Fourth, the RICH velocity resolution is $\Delta \beta / \beta=5 \times 10^{-4}$ [34] for carbon. The carbon rigidity resolution function up to $20 \mathrm{GV}$, including non-Gaussian tails, was obtained with data using the RICH only and found to be in good agreement with the rigidity resolution function from the simulation similar to Ref. [5].

The first three procedures provide the MDR of $3 \mathrm{TV}$ for boron and $2.6 \mathrm{TV}$ for carbon with $5 \%$ uncertainty. The second, third, and fourth verify the nucleus-nucleus elastic and quasielastic scattering in the AMS materials and provide the uncertainty of the amplitude of the nonGaussian tails in the rigidity resolution function to be $10 \%$.

The systematic error on the $\mathrm{B} / \mathrm{C}$ ratio due to the rigidity resolution functions was obtained by repeating the unfolding procedure varying the widths of the Gaussian core of resolution functions by $5 \%$ for both boron and carbon, and varying the amplitude of the non-Gaussian tails by $10 \%$. The resulting systematic error is less than $1 \%$ below $100 \mathrm{GV}$ and increasing to $5 \%$ at $2.6 \mathrm{TV}$.

There are two contributions to the systematic uncertainty on the $\mathrm{B} / \mathrm{C}$ ratio, from residual tracker misalignment and from magnetic field temperature correction, as discussed in Ref. [4]. The error is derived by simultaneously modifying the boron and carbon rigidity scales for alignment and magnetic field estimated uncertainties. The resulting error is $<1 \%$ below to $100 \mathrm{GV}$ and $2.5 \%$ at $2.6 \mathrm{TV}$.

To ensure that the treatment of systematic errors is correct an additional verification was performed. Figure 7 of the Supplemental Material [31] shows the ratio of two measurements of the $\mathrm{B} / \mathrm{C}$ ratio from $1.9 \mathrm{GV}$ to $0.88 \mathrm{TV}$ performed using events passing through $L 1$ to $L 8$ and using events passing through $L 1$ to $L 9$. The good agreement between the two measurements verifies (a) the systematic errors due to the difference in the resolution functions and (b) systematic errors on acceptance due to the difference in geometric factor and the amount of material traversed.

Most importantly, several independent analyses were performed on the same data sample by different study groups. The results of those analyses are consistent with this Letter. 
Results.-The measured $\mathrm{B} / \mathrm{C}$ ratio including statistical errors and systematic errors is tabulated in Table I of the Supplemental Material [31] as a function of the rigidity at the top of the AMS detector. The contributions to the systematic errors come from (i) the background subtraction, (ii) the trigger and the acceptance calculation, (iii) the unfolding procedure and the rigidity resolution function, (iv) the absolute rigidity scale. The contribution of individual sources to the systematic error are added in quadrature to arrive at the total systematic error. The Monte Carlo event samples have sufficient statistics such that they do not contribute to the errors.

Figure 1 shows the $\mathrm{B} / \mathrm{C}$ ratio as a function of rigidity with the total errors, the sum in quadrature of statistical and systematic errors.

The $\mathrm{B} / \mathrm{C}$ ratio increases with rigidity reaching a maximum at $4 \mathrm{GV}$ then decreases. The $\mathrm{B} / \mathrm{C}$ ratio does not show any significant structures. The current, more precise data will allow stringent limits to be placed on models that require such structures [6-8].

Above $65 \mathrm{GV}$ the $\mathrm{B} / \mathrm{C}$ ratio measured by AMS is well fit with a single power law $(\mathrm{B} / \mathrm{C})=k R^{\Delta}$, where $k$ is a constant normalization factor, with a $\chi^{2} /$ d.o.f. $=20 / 24$ and a spectral index $\Delta=-0.333 \pm 0.014$ (fit) \pm 0.005 (syst). The first error (fit) takes into account the statistical and uncorrelated systematic errors, the second (syst) is the error from the remaining systematic errors, namely, from the rigidity resolution function, rigidity scale, and boron and carbon inelastic cross sections, with proper accounting of the binto-bin correlations. We obtain a consistent value of $\Delta$ starting the fitting in the range from 50 to $80 \mathrm{GV}$. The measured $\Delta$ is in good agreement with the Kolmogorov theory of turbulence which predicts $\Delta=-1 / 3$ asymptotically $[1,2]$.

To obtain the variation of the spectral index $\Delta$ with rigidity in a model independent way, the spectral index is calculated from

$$
\Delta=d[\log (\mathrm{B} / \mathrm{C})] / d[\log (R)]
$$

over nonoverlapping rigidity intervals. The result of this fitting procedure is shown in Fig. 2.

To compare AMS results with previous measurements the following procedure to convert the $\mathrm{B} / \mathrm{C}$ ratio from rigidity to kinetic energy per nucleon $E_{K}$ is used. The kinetic energy per nucleon binning is derived from the rigidity binning using $E_{K}=\frac{1}{12}\left(\sqrt{(6 R)^{2}+M_{\mathrm{C}}^{2}}-M_{\mathrm{C}}\right)$ where $M_{\mathrm{C}}$ is the ${ }^{12} \mathrm{C}$ mass. The obtained binning was converted back into a new rigidity binning for the boron measurement using $\tilde{R}=\frac{1}{5} \sqrt{\left(A_{\mathrm{B}} E_{K}+M_{\mathrm{B}}\right)^{2}-M_{\mathrm{B}}^{2}}$ where $A_{\mathrm{B}}=10\left(1-Y_{\mathrm{B}}\right)+11 Y_{\mathrm{B}}$ and $M_{\mathrm{B}}=M_{{ }^{10} \mathrm{~B}}\left(1-Y_{\mathrm{B}}\right)+$ $M_{{ }_{11} \mathrm{~B}} Y_{\mathrm{B}}$, where $M_{{ }^{10} \mathrm{~B}}$ and $M_{{ }^{1{ }_{\mathrm{B}}}}$ are the ${ }^{10} \mathrm{~B}$ and ${ }^{11} \mathrm{~B}$ masses, and $Y_{\mathrm{B}}$ is the assumed boron isotopic composition $Y_{\mathrm{B}}=$ $0.7 \pm 0.1$ according to AMS low energy measurements [32]. The B/C ratio in Eq. (2) has been obtained using
$N_{i}^{\mathrm{B}}, A_{i}^{\mathrm{B}}$ and $\epsilon_{i}^{\mathrm{B}}$ calculated for each bin $\left(\tilde{R}_{i} ; \tilde{R}_{i}+\Delta \tilde{R}_{i}\right)$ and $N_{i}^{\mathrm{C}}, A_{i}^{\mathrm{C}}$, and $\epsilon_{i}^{\mathrm{C}}$ from the original rigidity binning $\left(R_{i} ; R_{i}+\Delta R_{i}\right)$. An additional systematic error due to the conversion procedure is derived varying the boron isotopic composition in the range $Y_{\mathrm{B}}=0.7 \pm 0.1$. This error is $1 \%$ at $1 \mathrm{GeV} / n$ and increases up to $4 \%$ at $1.3 \mathrm{TeV} / n$.

The $\mathrm{B} / \mathrm{C}$ ratio in kinetic energy per nucleon is presented in Table II of the Supplemental Material [31], including errors converted from corresponding errors in Table I of the Supplemental Material [31] with the additional error due to conversion. Figure 3 shows the AMS B/C ratio together with recent results. Also shown is the prediction for the $\mathrm{B} / \mathrm{C}$ ratio from an important theoretical model [7], which explains the AMS positron fraction [10] and antiproton results [11] by secondary production in cosmic ray propagation. The model shown is ruled out by this measurement.

In conclusion, the precise measurement of the boron to carbon flux ratio in cosmic rays with rigidity from $1.9 \mathrm{GV}$ to $2.6 \mathrm{TV}$ based on 2.3 million boron and 8.3 million carbon nuclei is presented. The $\mathrm{B} / \mathrm{C}$ ratio increases with rigidity reaching a maximum at $4 \mathrm{GV}$ then decreases. The $\mathrm{B} / \mathrm{C}$ ratio does not show any significant structures. The current, more precise data will allow stringent limits to be placed on models that require such structures [6-8]. Above $65 \mathrm{GV}$ the $\mathrm{B} / \mathrm{C}$ ratio can be described by a single power law of $\Delta=-0.333 \pm 0.014$ (fit) \pm 0.005 (syst), in good agreement with the Kolmogorov theory of turbulence which predicts $\Delta=-1 / 3$ asymptotically $[1,2]$.

We thank former NASA Administrator Daniel S. Goldin for his dedication to the legacy of the ISS as a scientific laboratory and his decision for NASA to fly AMS as a DOE payload. We also acknowledge the continuous support of the NASA leadership including Charles Bolden and William H. Gerstenmaier and of the JSC and MSFC flight control teams which has allowed AMS to operate optimally on the ISS for over five years. We are grateful for the support of Jim Siegrist and his staff of the DOE. We also acknowledge the continuous support from MIT and its School of Science, Michael Sipser, Marc Kastner, Ernest Moniz, Richard Milner, and Boleslaw Wyslouch. Research supported by São Paulo Research Foundation (FAPESP) Grants No. 2015/50378-5, No. 2015/13533-2, and No. 2016/10222-9, Brazil; CAS, NSFC, MOST, NLAA, the provincial governments of Shandong, Jiangsu, Guangdong, and the China Scholarship Council, China; action H2020 MSCA-IF-2015 under Grant No. 707543MAtISSE, European Union; the Finnish Funding Agency for Innovation (Tekes) Grants No. 40361/01 and No. 40518/03 and the Academy of Finland Grant No. 258963, Finland; CNRS/IN2P3, CNES, Enigmass, and the ANR, France; Pascale Ehrenfreund, DLR, and JARA-HPC under Project No. JARA0052, Germany; INFN and ASI under ASI-INFN Agreements No. 2013-002-R.0 and No. 2014-037-R.0, Italy; CHEP and NRF under Grants 
No. NRF-2009-0080142 and No. NRF-2012-010226 at Kyungpook National University and No. NRF-2013004883 at Ewha Womans University, Korea; the Consejo Nacional de Ciencia y Tecnología and UNAM, Mexico; FCT under Grant No. PTDC/FIS/122567/2010, Portugal; CIEMAT, IAC, CDTI, and SEIDI-MINECO under Grants No. AYA2012-39526-C02-(01/02), No. ESP2015-71662C2-(1-P/2-P), No. SEV-2011-0187, No. SEV-2015-0548, and No. MDM-2015-0509, Spain; the Swiss National Science Foundation (SNSF), federal and cantonal authorities, Switzerland; Academia Sinica and the Ministry of Science and Technology (MOST) under Grants No. 1032112-M-006-018-MY3, No. 105-2112-M-001-003, and No. CDA-105-M06, former President of Academia Sinica Yuan-Tseh Lee, and former Ministers of MOST Maw-Kuen $\mathrm{Wu}$ and Luo-Chuan Lee, Taiwan; the Turkish Atomic Energy Authority at METU, Turkey; and NSF Grants No. 1455202 and No. 1551980, Wyle Laboratories Grant No. 2014/T72497, and NASA NESSF Grant No. HELIO15F-0005, USA. We gratefully acknowledge the strong support from CERN including Rolf-Dieter Heuer and Fabiola Gianotti, from the CERN IT department and Bernd Panzer-Steindel, and from the European Space Agency including Johann-Dietrich Wörner and Simonetta Di Pippo. We are grateful for important physics discussions with Fiorenza Donato, Jonathan Ellis, Jonathan Feng, Igor Moskalenko, Michael Salamon, Subir Sarkar, Joachim Trümper, Michael S. Turner, Steven Weinberg, and Arnold Wolfendale.

${ }^{a}$ Also at ASI, I-00133, Roma, Italy.

${ }^{\mathrm{b}}$ Also at ASI Science Data Center (ASDC), I-00133, Roma, Italy.

${ }^{c}$ Also at Sun Yat-Sen University (SYSU), Guangzhou, 510275, China.

${ }^{\mathrm{d}}$ Also at Wuhan University, Wuhan, 430072, China.

eAlso at Harbin Institute of Technology (HIT), Harbin, 150001, China.

${ }^{\mathrm{f}}$ Also at Huazhong University of Science and Technology (HUST), Wuhan, 430074, China.

${ }^{\mathrm{g}}$ Also at Università di Siena, I-53100, Siena, Italy.

${ }^{\mathrm{h}}$ Also at Laboratoire d'Annecy-le-Vieux de Physique Théorique (LAPTh), CNRS and Université Savoie Mont Blanc, F-74941 Annecy-le-Vieux, France.

${ }^{\mathrm{i}}$ Also at Shandong University (SDU), Jinan, Shandong, 250100, China.

${ }^{\mathrm{j}}$ Also at Nankai University, Tianjin, 300071, China.

${ }^{\mathrm{k}}$ Also at Southeast University (SEU), Nanjing, 210096, China.

${ }^{1}$ Also at Institute of Theoretial Physics, Chinese Academy of Sciences, Beijing, 100190, China.

${ }^{\mathrm{m}}$ Also at Jilin University, Jilin, 130012, China.

[1] I. A. Grenier, J. H. Black, and A. W. Strong, Annu. Rev. Astron. Astrophys. 53, 199 (2015); P. Blasi, Astron. Astrophys. Rev. 21, 70 (2013); A. W. Strong, I. V. Moskalenko, and V.S. Ptuskin, Annu. Rev. Nucl. Part.
Sci. 57, 285 (2007); A. Castellina and F. Donato, Astropart. Phys. 24, 146 (2005).

[2] A. N. Kolmogorov, Dokl. Akad. Nauk SSSR 30, 301 (1941); Proc. R. Soc. A 434, 9 (1991).

[3] R. H. Kraichnan, Phys. Fluids 8, 1385 (1965).

[4] M. Aguilar et al., Phys. Rev. Lett. 114, 171103 (2015).

[5] M. Aguilar et al., Phys. Rev. Lett. 115, 211101 (2015).

[6] N. Tomassetti, Phys. Rev. D 92, 081301(R) (2015); for earlier work see, A. E. Vladimirov, G. Jóhannesson, I. V. Moskalenko, and T. A. Porter, Astrophys. J. 752, 68 (2012).

[7] R. Cowsik, B. Burch, and T. Madziwa-Nussinov, Astrophys. J. 786, 124 (2014); R. Cowsik, Annu. Rev. Nucl. Part. Sci. 66, 297 (2016); R. Cowsik and T. Madziwa-Nussinov, Astrophys. J. 827, 119 (2016).

[8] P. Mertsch and S. Sarkar, Phys. Rev. D 90, 061301(R) (2014).

[9] I. Cholis and D. Hooper, Phys. Rev. D 89, 043013 (2014).

[10] L. Accardo et al., Phys. Rev. Lett. 113, 121101 (2014).

[11] M. Aguilar et al., Phys. Rev. Lett. 117, 091103 (2016).

[12] C. D. Orth, A. Buffington, G. F. Smoot, and T. S. Mast, Astrophys. J. 226, 1147 (1978); R. Dwyer and P. Meyer, Astrophys. J. 322, 981 (1987); M. Simon, H. Spiegelhauer, W. K. H. Schmidt, F. Siohan, J. F. Ormes, V. K. Balasubrahmanyan, and J. F. Arens, Astrophys. J. 239, 712 (1980).

[13] J. J. Engelmann et al., Astron. Astrophys. 233, 96 (1990).

[14] W. R. Webber et al., Proceedings of the 19th International Cosmic Ray Conference, La Jolla, CA (Scientific and Technical Information Branch, NASA, Washington, 1985), Vol. 2, p. 16.

[15] D. Muller, S. P. Swordy, P. Meyer, J. L'Heureux, and J. M. Grunsfeld, Astrophys. J. 374, 356 (1991).

[16] J. Buckley, J. Dwyer, D. Mueller, S. Swordy, and K. K. Tang, Astrophys. J. 429, 736 (1994).

[17] M. Aguilar et al., Astrophys. J. 724, 329 (2010).

[18] A. D. Panov et al., Proceedings of the 30th International Cosmic Ray Conference, Mérida (Universidad Nacional Autónoma de México, Mexico City, 2008), Vol. 2, p. 3.

[19] H. S. Ahn et al., Astropart. Phys. 30, 133 (2008).

[20] A. Obermeier, P. Boyle, J. Hörandel, and D. Müller, Astrophys. J. 752, 69 (2012).

[21] O. Adriani et al., Astrophys. J. 791, 93 (2014).

[22] A. Kounine, Int. J. Mod. Phys. E 21, 1230005 (2012); S. Rosier-Lees, in Proceedings of Astroparticle Physics TEVPA/IDM, Amsterdam, 2014 (to be published); S. Ting, Nucl. Phys. B, Proc. Suppl. 243-244, 12 (2013); S.-C. Lee, Proceedings of the 20th International Conference on Supersymmetry and Unification of Fundamental Interactions (SUSY 2012), Beijing, 2012 (unpublished); M. Aguilar, Proceedings of the XL International Meeting on Fundamental Physics, Centro de Ciencias de Benasque Pedro Pascual, 2012 (unpublished); S. Schael, Proceedings of the 10th Symposium on Sources and Detection of Dark Matter and Dark Energy in the Universe, Los Angeles, 2012 (unpublished); B. Bertucci, Proc. Sci., EPS-HEP (2011) 67; M. Incagli, AIP Conf. Proc. 1223, 43 (2010); R. Battiston, Nucl. Instrum. Methods Phys. Res., Sect. A 588, 227 (2008).

[23] K. Lübelsmeyer et al., Nucl. Instrum. Methods Phys. Res., Sect. A 654, 639 (2011). 
[24] B. Alpat et al., Nucl. Instrum. Methods Phys. Res., Sect. A 613, 207 (2010).

[25] V. Bindi et al., Nucl. Instrum. Methods Phys. Res., Sect. A 743, 22 (2014), and references therein.

[26] J. Allison et al., IEEE Trans. Nucl. Sci. 53, 270 (2006); S. Agostinelli et al., Nucl. Instrum. Methods Phys. Res., Sect. A 506, 250 (2003).

[27] A. Boudard, J. Cugnon, J.-C. David, S. Leray, and D. Mancusi, Phys. Rev. C 87, 014606 (2013); S. Leray, D. Mancusi, P. Kaitaniemi, J. C. David, A. Boudard, B. Braunn, and J. Cugnon, J. Phys. Conf. Ser. 420, 012065 (2013).

[28] J. Ranft, Phys. Rev. D 51, 64 (1995).

[29] J. Alcaraz et al., Phys. Lett. B 484, 10 (2000).

[30] C. C. Finlay et al., Geophys. J. Int. 183, 1216 (2010); E. Thébault et al., Earth Planets Space 67, 79 (2015).

[31] See Supplemental Material at http://link.aps.org/ supplemental/10.1103/PhysRevLett.117.231102 for supporting figures and the tabulated $\mathrm{B} / \mathrm{C}$ flux ratio in rigidity and kinetic energy per nucleon.

[32] AMS Collaboration, Measurement of the Flux of Light Nuclei in Primary Cosmic Rays with the Alpha Magnetic Spectrometer on the International Space Station (to be published).

[33] J. Jaros et al., Phys. Rev. C 18, 2273 (1978); V. D. Aksinenko et al., Nucl. Phys. A348, 518 (1980); S. Kox, A. Gamp, R. Cherkaoui, A. J. Cole, N. Longequeue, J. Menet, C. Perrin, and J. B. Viano, Nucl. Phys. A420, 162 (1985); S. Kox et al., Phys. Rev. C 35, 1678 (1987); D. Q. Fang et al., Phys. Rev. C 61, 064311 (2000); A. Ozawa et al., Nucl. Phys. A691, 599 (2001); H. Y. Zhang et al., Nucl. Phys. A707, 303 (2002); T. Zheng et al., Nucl. Phys. A709, 103 (2002); M. Takechi et al., Phys. Rev. C 79, 061601 (2009).

[34] F. Giovacchini, Nucl. Instrum. Methods Phys. Res., Sect. A 766, 57 (2014). 